\title{
BMJ Open Mapping the global evidence on nutrition transition: a scoping review protocol
}

\author{
Jessica Emily Singh, ${ }^{1,2}$ Anne-Kathrin Illner, ${ }^{3}$ Klara Dokova, ${ }^{4}$ Natalya Usheva, ${ }^{5}$ \\ Todorka Kostadinova, ${ }^{6}$ Krasimira Aleksandrova (i) ${ }^{1,7}$
}

To cite: Singh JE, IIIner A-K, Dokova $\mathrm{K}$, et al. Mapping the global evidence on nutrition transition: a scoping review protocol. BMJ Open 2020;10:e034730. doi:10.1136/ bmjopen-2019-034730

- Prepublication history for this paper is available online To view these files, please visit the journal online (http://dx.doi org/10.1136/bmjopen-2019034730).

Received 03 October 2019 Revised 09 March 2020 Accepted 03 April 2020
Check for updates

(C) Author(s) (or their employer(s)) 2020. Re-use permitted under CC BY-NC. No commercial re-use. See rights and permissions. Published by BMJ.

For numbered affiliations see end of article.

Correspondence to Dr Krasimira Aleksandrova; krasimira.aleksandrova@dife.de

\section{ABSTRACT}

Introduction Nutrition transition has emerged as an important concept in health research used to describe shifts in dietary consumption and energy expenditure that coincide with economic, demographic and epidemiological changes at a population level. Better understanding of the shifts in dietary patterns across populations and their drivers could possibly hold the key to prevention of dietrelated disease risk. An increasing number of studies have reported on nutrition transition in populations around the world, however, global evidence has not been summarised. Objective This scoping review is aimed to identify, explore and map the literature on nutrition transition with a specific focus on dietary changes in populations across the world. The review would allow better clarification around the concept of nutrition transition, classification of published studies and provide a framework for further research.

Methods and analysis The scoping review will be designed based on the methodology by Arksey and 0 'Malley, refined by Levac et al. and developed in conjunction with guidance on conducting systematic scoping reviews by Peters et al. The main research question addressed by the scoping review will be: 'What is the evidence on nutrition transition defined based on dietary changes reported in general adult population across the world?' Electronic databases (PubMed, ScienceDirect and Web of Science), grey literature sources and the reference lists of key studies will be searched to identify studies appropriate for inclusion in the review. Two reviewers will independently screen all abstracts and full-text studies for inclusion. Data will be abstracted into tables and logically organised according to items addressed in the specific research questions.

Ethics and dissemination Dissemination of results will be sought through a peer-reviewed publication, conference presentations and stakeholder meetings. The data used are from publicly available secondary sources, so no ethical review would be required for this study.

\section{BACKGROUND}

Suboptimal diet has long been established as one of the leading contributors to the global burden of disease (GBD). It has been estimated that one in five deaths globally, equivalent to 11 million deaths, are associated with suboptimal diet. ${ }^{1}$ In 2017, more deaths were

\section{Strengths and limitations of this study}

This will be the first scoping review on the topic of nutrition transition with a special focus on dietary changes and associated driving factors, collating and summarising relevant published scientific evidence in a worldwide context.

- The search strategy includes electronic databases with peer-reviewed literature, also incorporating article bibliographies and grey literature sources.

- This scoping review is expected to provide an overview of the overall findings on the nutrition transition defined based on dietary changes reported in general adult population represented by different countries/continental regions across the world and to issue recommendations for future research.

- This scoping review will focus on a general adult population, hence studies including children and adolescents will be excluded.

caused by diets characterised by low intakes of foods such as whole grains, fruit, nuts and seeds than by diets high in trans-fats, sugary drinks and red and processed meat intakes. ${ }^{23}$

Dietary patterns have never been constant and have been prone to changes due to a variety of factors including climate, geographical location, politics, cultural influences and religious practices. ${ }^{45}$ As changes occur at differing rates across the world, adaptation of the diet over time has been also heterogeneous. In this context, the term 'nutrition transition' was coined by Popkin in the 1990s to describe the shifts in dietary consumption and energy expenditure that coincide with economic, demographic and epidemiological changes. ${ }^{6}$ According to Popkin, human nutrition has undergone several transition patterns since the dawn of mankind, summarised as follows: (1) a hunter-gatherer, labourintensive lifestyle, with diets high in carbohydrates and low in saturated fat; (2) initial settlement lifestyle, with diets dominated by cereals and emergence of nutritional deficiencies; (3) a low-fat, high-fibre diet with an 
increase in the consumption of fruits, vegetables, animal protein and a decrease in starchy staples; (4) diets high in fat, sugar, refined carbohydrates and processed foods, and reduced physical activity and (5) intentional reduction in calorie and fat intake and increased consumption of fruits, vegetables, unrefined carbohydrates accompanied with a higher level of physical activity. ${ }^{7}$

Overall, the theory of nutrition transition proposes that with urbanisation, economic development and technological advancement, traditional diets have been substituted by diets high in vegetable oils, sugar, refined carbohydrates and animal-sourced foods. ${ }^{8}$ For example, increased processed and ultra-processed food consumption and reduced food biodiversity have emerged as key dietary changes that describe the nutrition transition phenomenon. ${ }^{9}$ Recently, the use of the term 'nutrition transition' in the literature has shown an immense increase. ${ }^{5}$ This term has been predominantly used to denote the transitions observed in developing countries from traditional diets high in cereal and fibre to more Western pattern diets high in sugars, fat and animalsource food. ${ }^{310}$ Numerous studies on nutrition transition have been published reporting on dietary changes in populations both from low-income and middle-income countries and high-income countries. ${ }^{11-16}$ Shifting dietary patterns have been found to largely coincide with rising rates of obesity, ${ }^{17}$ diabetes ${ }^{18}$ and overall chronic diseases. ${ }^{19}$ Better understanding of the shifts in dietary patterns across populations and their drivers could possibly hold the key to prevention of diet-related disease risk. ${ }^{19}$

While several reviews on nutrition transition have been previously published, these have either focused on evidence from specific countries ${ }^{20}$ or continental regions. ${ }^{21}$ The global evidence on nutrition transition has not been summarised so far. Moreover, nutrition transition is a term used to identify a complex phenomenon and the key concepts and definitions used in the literature require better clarification. Especially, it remains important to evaluate how research on nutrition transition has been conducted, that is, which methods have been used to measure dietary changes over time and to classify individual-based versus population-based approaches for data collection. ${ }^{22}$ In this context, we propose conducting a scoping review that would allow better clarification around the concept of nutrition transition, classification of published studies and provide a framework for further research. $^{23}$

\section{STUDY RATIONALE}

This scoping review will be the first, to our knowledge, to assess the literature published on the topic of nutrition transition worldwide. An overview of the research on a global scale would provide an important basis for understanding how nutrition transition was studied, to classify published studies and to identify existing gaps in research. The results of this scoping review will essentially provide a framework for conducting research on nutrition transition. Ultimately, such research will bring important insights for guiding strategies aimed at reducing dietrelated chronic disease burden.

\section{STUDY OBJECTIVES}

The main objective for the proposed scoping review is to identify, explore and map the literature on nutrition transition defined based on dietary changes reported in general adult populations across the world.

\section{METHODS AND ANALYSIS}

Prior to designing the protocol for this scoping review, literature on the nutrition transition was first searched through PubMed for any prior scoping reviews published in the field. No previously published scoping reviews on this topic were found. PROSPERO was also checked for any registered scoping reviews yet to be conducted, with no scoping reviews on nutrition transition registered. The literature search started on 1 August 2019 and ended on 15 December 2019.

\section{Protocol design}

The methods for this scoping review are based on Arksey and O'Malley's scoping review methodology, ${ }^{24}$ further refined by Levac et $a l^{25}$ and also considering a guidance for conducting systematic scoping reviews by Peters et $a l^{26}$ The protocol is based around six fundamental stages: (1) identifying research questions; (2) identifying relevant studies; (3) selection of eligible studies; (4) data abstraction and charting of results; (5) data summary and synthesis of results and (6) consultation with relevant stakeholders. These six stages are further detailed below.

\section{Box 1 Review research questions}

Research question (considering population, concept, context) What is the evidence on nutrition transition defined based on dietary changes reported in general adult populations across the world? Other research questions

What are the different nutrition transition patterns reported on in adult population?

Are there countries/continental region-specific dietary patterns consistently reported?

What are the main sources for data generated?

What are the most frequently used methods for dietary data collection (population-based vs individual-based)?

Are there underrepresented countries/continental regions in published research on the nutrition transition?

What are the potential factors/drivers of dietary change associated with nutrition transition previously identified?

What are coinciding health and disease patterns associated with these dietary changes?

What are the main gaps in the literature and directions for future research? 
Stage 1: identifying the research questions

The review questions given in box 1 were defined as a result of a brief search on the scope of published evidence on the nutrition transition and based on discussions with the research team. The main research question takes into account 'population', 'concept' and 'context' as outlined below.

\section{Population}

The selected populations will be adults, 18 years and older (not pregnant, not breastfeeding women), assessed when this information is stated.

\section{Concept}

This scoping review will focus on dietary changes as a core aspect of nutrition transition. Studies reporting on other behaviours, such as physical activity or health outcomes, that is, body mass index, will not be included in the review.

\section{Context}

The context will be review of data collected on dietary changes over time reported as part of the nutrition transition.

\section{Stage 2: identifying relevant studies}

Discussions between authors led to agreement on the eligibility criteria, online databases to be searched, determination of key terms and the search strategy. No timeframe restrictions for studies were agreed.

\section{Eligibility criteria}

In order to determine the eligibility criteria, the 'population', 'concept' and 'context' as outlined in Stage 1 were considered.

\section{Inclusion and exclusion criteria and rationale}

The inclusion and exclusion criteria are given in table 1 in conjunction with the rationale behind each criterion.

\section{Search strategy}

A three-step method will be followed, as recommended by Peters et al..$^{26}$ Published and unpublished (grey literature) will be searched using several online databases, and identified literature will be screened based on title, abstracts and key terms. The search terms included 'nutrition transition' or 'nutrition transitions' or 'nutritional transition' or 'nutritional transitions'. After that, a full assessment of papers which meet the inclusion and exclusion criteria will be conducted. Reference lists of all included literature and excluded reviews will be additionally searched for any further relevant literature. Only articles in English will be included in the scoping review. However, to allow appraisal of possible publication bias, identified articles that have been published in languages other than English will be counted. No limitations will be set for the publication period.

\section{Databases}

The search will be conducted within three large online databases (PubMed, ScienceDirect and Web of Science). Endnote V. X7 will be used as the reference management software to assist in the organisation and abstraction of data.

\section{Stage 3: selection of eligible studies}

The study selection method will be as above to identify potential publications, an elimination process will be conducted (based on title, abstract, key terms, full articles in ascending order) by two independent researchers.

Table 1 Inclusion and exclusion criteria for eligible studies

\begin{tabular}{ll}
\hline Study inclusion criteria & Rationale \\
\hline $\begin{array}{l}\text { Written in English } \\
\text { Includes data on dietary intake or dietary }\end{array}$ & $\begin{array}{l}\text { To include studies targeted at an international audience. } \\
\text { The term nutrition transition encompasses a number of factors; however, dietary } \\
\text { change is the primary factor. Behaviours, such as food purchasing trends, will also } \\
\text { be captured. }\end{array}$ \\
$\begin{array}{l}\text { Reports on more than one time-point } \\
\text { In order for studies to assess change in diet, it was decided that only studies } \\
\text { reporting evidence for more than one time-point be included. }\end{array}$ \\
$\begin{array}{l}\text { Reports on adult population (18 years and } \\
\text { older) }\end{array}$ & $\begin{array}{l}\text { To allow better understanding of the shifts in dietary patterns across populations at } \\
\text { higher chronic disease risk only adult and older age groups will be included in the } \\
\text { review. } \\
\text { In order to allow generalisibility of results, we will include studies based on } \\
\text { representative samples of the population of interest. }\end{array}$ \\
$\begin{array}{l}\text { Reviews analysing and reporting on secondary } \\
\text { data }\end{array}$ & $\begin{array}{l}\text { Reviews that incorporate secondary data on changes of diet over time taken from } \\
\text { public databases (eg, FAOSTAT) will be included in the review. }\end{array}$ \\
$\begin{array}{l}\text { Study exclusion criteria } \\
\text { Reviews with no secondary quantitative data }\end{array}$ & $\begin{array}{l}\text { Studies of a narrative style review with no secondary quantitative data will be } \\
\text { excluded, as a quantitative comparison will be conducted. Reference lists from } \\
\text { these reviews will be examined. }\end{array}$ \\
\hline $\begin{array}{l}\text { To avoid duplication of data, papers with no original quantitative diet data will be } \\
\text { excluded. }\end{array}$
\end{tabular}


Study selection will be completed using a web-based software (Covidence), which facilitates screening, study selection and data extraction for literature and systematic reviews and is recommended by Cochrane. ${ }^{27}$ The results were compared and consolidated through consensus between the two researchers in November 2019. The final list of included articles was further reviewed by the senior author in December 2019. Any disagreement on the final papers to be included will be discussed until an agreement is reached by all authors. The number of included and excluded articles will be presented in a search decision flowchart as outlined in the Preferred Reporting Items for Systematic Reviews and Meta-Analyses guidelines ${ }^{28}$ and the search decision process reported in a narrative form also.

\section{Stage 4: data abstraction and charting of results}

Two researchers will independently conduct the data abstraction once studies for inclusion have been agreed on, in January 2020. In the instance of any discrepancies in data abstracted, these will be further assessed by the senior author to ensure reaching a consensus on study inclusion. This data will be charted in a table including, but not limited to, the following: region, study design, country, authors, year published, number of participants, duration of study period, methodology for diet data collection, main findings and limitations. The data will be categorised based on the suggestion by the WHO GBD regional classification system: African Region, Eastern Mediterranean Region, European Region, Region of the Americas, South-East Asian Region and Western Pacific Region. This data will then be used for the synthesis of findings in a narrative form.

\section{Stage 5: data summary and synthesis of results}

The aggregated findings will provide an overview of the research conducted on nutrition transition worldwide, rather than an assessment of the quality of all individual studies. Data will be summarised both quantitatively (eg, proportion of region covered based on population, total years covered within countries/regions studied) and qualitatively (eg, overall summary of evidence, findings, methodological approaches and limitations). The key characteristics and concepts of the articles collected in the data extraction table will be identified and summarised. The synthesis of results may be further developed based on the findings of the scoping review, in order to best capture the key characteristics and concepts of the published literature.

\section{Stage 6: consultation with relevant stakeholders}

Relevant stakeholders will be consulted during the conducting of the scoping review. Specifically, they will provide input regarding the search strategy to ensure data collected is representative of the true nature of the field. Stakeholders will include experts on the subject of nutrition transition. These stakeholders will be identified through their relevant publications across the regions.

\section{ETHICS AND DISSEMINATION}

Results from this scoping review will be published in a relevant peer-reviewed journal and presented at conferences. Ethics are not required due to the nature of the study, based on collection of publicly available data.

\section{PATIENT AND PUBLIC INVOLVEMENT}

No participants (patients or public) will be involved.

\section{CONCLUSIONS}

Since the definition of the term nutrition transition, many papers have been published worldwide trying to capture the progression of dietary changes in response to drivers such as globalisation, modernisation and affluence. Despite the large number of publications on the topic, a scoping review has not yet been published on the nutrition transition and hence, here we propose a protocol for conducting a scoping review aimed to summarise overall findings on the global nutrition transition. Ultimately, we hope that our work will lead the way for valuable future contributions to the field of nutrition transition.

\section{Author affiliations \\ ${ }^{1}$ Senior Scientist Group Nutrition, Immunity and Metabolism, Department of Nutrition and Gerontology, German Institute of Human Nutrition Potsdam- \\ Rehbrücke, Nuthetal, Potsdam-Rehbrücke, Germany \\ ${ }^{2}$ Department of Dietetics, Nutrition and Sport, La Trobe University, Melbourne, Victoria, Australia \\ ${ }^{3}$ College of Health Sciences, UniLaSalle, Beauvais, France \\ ${ }^{4}$ Faculty of Public Health, Medical University of Varna, Varna, Bulgaria \\ ${ }^{5}$ Department of Social Medicine and Health Care Organization, Medical University of Varna, Varna, Bulgaria \\ ${ }^{6}$ Department of Health Economics and Management, Medical University of Varna, Varna, Bulgaria \\ ${ }^{7}$ Institute of Nutritional Science, University of Potsdam, Potsdam, Germany}

Contributors KA, A-KI, KD, NU, TK worked together to obtain funding to undertake this collaborative work. All authors helped conceptualise the study protocol for the scoping review through discussions on the topic. JES and KA wrote the manuscript, with feedback provided by A-KI, TK, KD, NU. All authors read and approved the final version of the manuscript that was submitted for publication.

Funding This work was supported by The German Federal Ministry of Education and Research (BMBF) (Grant No. 01DS19002).

Competing interests None declared.

Patient consent for publication Not required.

Provenance and peer review Not commissioned; externally peer reviewed.

Open access This is an open access article distributed in accordance with the Creative Commons Attribution Non Commercial (CC BY-NC 4.0) license, which permits others to distribute, remix, adapt, build upon this work non-commercially, and license their derivative works on different terms, provided the original work is properly cited, appropriate credit is given, any changes made indicated, and the use is non-commercial. See: http://creativecommons.org/licenses/by-nc/4.0/.

ORCID iD

Krasimira Aleksandrova http://orcid.org/0000-0002-1275-1827

\section{REFERENCES}

1 Forouhi NG, Unwin N. Global diet and health: old questions, fresh evidence, and new horizons. Lancet 2019;393:1916-8. 
2 GBD 2017 Diet Collaborators. Health effects of dietary risks in 195 countries, 1990-2017: a systematic analysis for the global burden of disease study 2017. Lancet 2019;393:1958-72.

3 Hawkes C. Uneven dietary development: linking the policies and processes of globalization with the nutrition transition, obesity and diet-related chronic diseases. Global Health 2006;2:4.

4 Popkin BM. Understanding the nutrition transition. Urban Health News/ 1996;30:3-19.

5 Popkin BM. Relationship between shifts in food system dynamics and acceleration of the global nutrition transition. Nutr Rev 2017;75:73-82.

6 Popkin BM. Nutritional patterns and transitions. Popul Dev Rev 1993;19:138-57.

7 Popkin BM. Global nutrition dynamics: the world is shifting rapidly toward a diet linked with noncommunicable diseases. Am J Clin Nutr 2006;84:289-98.

8 Popkin BM. An overview on the nutrition transition and its health implications: the Bellagio meeting. Public Health Nutr 2002;5:93-103.

9 Popkin BM. Nutrition in transition: the changing global nutrition challenge. Asia Pac J Clin Nutr 2001;10 Suppl:S13-18.

10 Fox A, Feng W, Asal V. What is driving global obesity trends? Globalization or "modernization"? Global Health 2019;15:32.

11 Du S, Lu B, Zhai F, et al. A new stage of the nutrition transition in China. Public Health Nutr 2002;5:169-74.

12 Misra A, Singhal N, Sivakumar B, et al. Nutrition transition in India: secular trends in dietary intake and their relationship to diet-related non-communicable diseases. J Diabetes 2011;3:278-92.

13 Moreno LA, Sarría A, Popkin BM. The nutrition transition in Spain: a European Mediterranean country. Eur J Clin Nutr 2002;56:992-1003.

14 Naughton SS, Mathai ML, Hryciw DH, et al. Australia's nutrition transition 1961-2009: a focus on fats. Br J Nutr 2015;114:337-46.

15 Shetty PS. Nutrition transition in India. Public Health Nutr 2002;5:175-82.
16 Zhai F, Wang H, Du S, et al. Prospective study on nutrition transition in China. Nutr Rev 2009;67 Suppl 1:S56-61.

17 Popkin BM, Adair LS, Ng SW. Global nutrition transition and the pandemic of obesity in developing countries. Nutr Rev 2012;70:3-21.

18 Popkin BM. Nutrition transition and the global diabetes epidemic. Curr Diab Rep 2015;15:64.

19 Astrup A, Dyerberg J, Selleck M, et al. Nutrition transition and its relationship to the development of obesity and related chronic diseases. Obes Rev 2008;9 Suppl 1:48-52.

20 Mehboob B, Safdar NF, Zaheer S. Socio-Economic, environmental and demographic determinants of rise in obesity among Pakistani women: a systematic review. J Pak Med Assoc 2016;66:1165-72.

21 Charlton KE, Russell J, Gorman E, et al. Fish, food security and health in Pacific island countries and territories: a systematic literature review. BMC Public Health 2016;16:285.

22 Walls HL, Johnston D, Mazalale J, et al. Why we are still failing to measure the nutrition transition. BMJ Glob Health 2018;3:e000657.

23 Munn Z, Peters MDJ, Stern C, et al. Systematic review or scoping review? guidance for authors when choosing between a systematic or scoping review approach. BMC Med Res Methodol 2018;18:143.

24 Arksey H, O'Malley L. Scoping studies: towards a methodological framework. Int J Soc Res Methodol 2005;8:19-32.

25 Levac D, Colquhoun H, O'Brien KK. Scoping studies: advancing the methodology. Implement Sci 2010;5:69.

26 Peters MDJ, Godfrey CM, Khalil H, et al. Guidance for conducting systematic scoping reviews. Int J Evid Based Healthc 2015;13:141-6.

27 Cochrane. Covidence, 2015. Available: https://community.cochrane. org/help/tools-and-software/covidence

28 Moher D, Liberati A, Tetzlaff J, et al. Preferred reporting items for systematic reviews and meta-analyses: the PRISMA statement. PLoS Med 2009;6:e1000097. 